\title{
Effects of an eight-week supervised, structured lifestyle modification programme on anthropometric, metabolic and cardiovascular risk factors in severely obese adults.
}

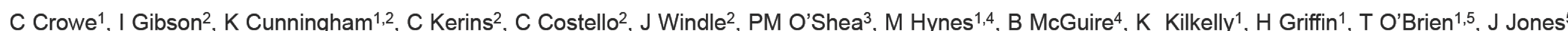
F M Finucane ${ }^{1,5}$.

${ }^{1}$ Bariatric Medicine Service, Galway Diabetes Research Centre, HRB Clinical Research Facility, Ireland.

${ }^{2}$ Croi, the West of Ireland Cardiac Foundation, Heart and Stroke Centre, Moyola Lane, Newcastle, Galway, Ireland.

${ }^{3}$ Department of Clinical Biochemistry, Galway University Hospitals, Galway, Ireland.

4 School of Psychology, National University of Ireland, Galway, Ireland.

${ }^{5}$ Discipline of Health Promotion, National Institute of Preventive Cardiology, National University of Ireland, Galway, Ireland.

\section{Introduction}

Lifestyle modification is fundamental to obesity treatment but few studies have described the effects of a structured lifestyle programme specifically in bariatric patients. We sought to describe changes in anthropometric and metabolic characteristics in a cohort of bariatric patients after participation in a nurse led, structured lifestyle programme. The eight-week programme was delivered over two hours each week. It focused on weight management as part of a comprehensive cardiovascular risk reduction strategy. The main emphasis of the programme was on lifestyle modification, namely healthy food choices, weight reduction, physical activity and smoking cessation.

\section{Methods}

We conducted a retrospective, observational cohort study of adults with a body mass index (BMI) $\geq 40 \mathrm{kgm}-2$ (or $\geq 35 \mathrm{kgm}-2 \mathrm{with}$ significant co-morbidity) who successfully participated in the progamme. Each patient was initially referred to the programme after careful assessment for suitability by the hospital-based, multidisciplinary bariatric team. Weight, height, waist circumference, blood pressure, $\mathrm{HbA1c}$, fasting glucose and lipid profiles as well as functional capacity (Incremental Shuttle Walk Test) and questionnairebased anxiety and depression scores before and after the programme were compared in per-protocol analyses.

\section{Results}

Of 183 bariatric patients enrolled, $150(81.9 \%)$ patients completed the programme. Mean age of completers was $47.9 \pm 11.2$ years. $34.7 \%$ were male. There were statistically significant reductions in weight, BMI, waist circumference (table 1) as well as anxiety and depression scores, totaland LDL-cholesterol and triglyceride levels, with an increase in functional capacity in completers at the end of the programme compared to enrolment into the programme. Blood pressure improved, with reductions in systolic and diastolic blood pressure, The proportion of patients achieving target blood pressure increased from 50.3 to $59.3 \%(p=0.04)$. The proportion of patients with diabetes achieving $\mathrm{HbA} 1 \mathrm{c}<53 \mathrm{mmol} / \mathrm{mol}$ increased from 28.6 to $42.9 \%,(p=0.02)$.

\begin{tabular}{|c|c|c|c|}
\hline & Enrolment & Completion & p Value \\
\hline Weight (kg) & $129.6 \pm 25.9$ & $126.9 \pm 26.1$ & $p<0.001$ \\
\hline BMI (kgm-2) & $46.3 \pm 8.3$ & $44.9 \pm 9.0$ & $p<0.001$ \\
\hline Waist women $(\mathrm{cm})$ & $133.0 \pm 17.1$ & $129.3 \pm 17.5$ & $p<0.001$ \\
\hline Waist men (cm) & $143.8 \pm 19.0$ & $135.1 \pm 17.9$ & $p<0.001$ \\
\hline $\begin{array}{l}\text { Functional capacity } \\
\text { (Mets) }\end{array}$ & $5.9 \pm 1.7$ & $6.8 \pm 2.1$ & $p<0.001$ \\
\hline SBP (mmHg) & $135 \pm 16.2$ & $131.6 \pm 17.1$ & $p<0.009$ \\
\hline DBP (mmHg) & $84.7 \pm 10.2$ & $81.4 \pm 10.9$ & $p<0.001$ \\
\hline
\end{tabular}

\section{Summary and Conclusion}

These findings confirm that a structured lifestyle modification programme for carefully selected bariatric patients is feasible and safe. Additionally it leads to statistically significant improvements in several cardiovascular risk-related outcomes. The retention rate of $81.9 \%$ compares favorably with similar lifestyle intervention studies in this patient group [1,2]. Although there were relatively small changes in body weight and BMl, fitness is a far more relevant index of overall wellness and cardiovascular risk than fatness. The mean increase of $15 \%$ in aerobic capacity (estimated MET max) of completers was a strong indicator that the programme is in fact effective and if sustained, might lead to a substantial reduction in mortality [3,4]. The improvements in depression scores and components of the lipid profile are also clearly relevant and important.

In conclusion, bariatric patients completing an eight week, nurse-led structured lifestyle programme had improved adiposity, fitness, lipid profiles, psychosocial health, blood pressure and glycaemia. Given that the programme appears feasible, safe and acceptable to patients, with a high retention rate and equivalent improvements to similar interventions in other cohorts of high risk individuals, future studies assessing the impact of structured lifestyle modification in bariatric patients could use it as a framework in a pragmatic randomised controlled trial.

\section{References}

(1) Ryan DH et al. Nonsurgical weight loss for extreme obesity in primary care settings: results of the Louisiana Obese Subjects Study. Arch Intern Med. 2010;170(2):146-54. doi:10.1001/archinternmed.2009.508.

(2) Goodpaster BH et al. Effects of diet and physical activity interventions on weight loss and cardiometabolic risk factors in severely obese adults: a randomized trial. Jama. 2010;304(16):1795-802. doi:10.1001/jama.2010.1505

(3) Gulati M et al. Exercise capacity and the risk of death in women: the St James Women Take Heart Project. Circulation. 2003;108(13):1554-9. doi

10.1161/01.CIR.0000091080.57509.E9.

(4) Myers et al . Exercise capacity and mortality among men referred for exercise testing. N Engl J Med. 2002;346(11):793-801. doi:10.1056/ NEJMoa011858.

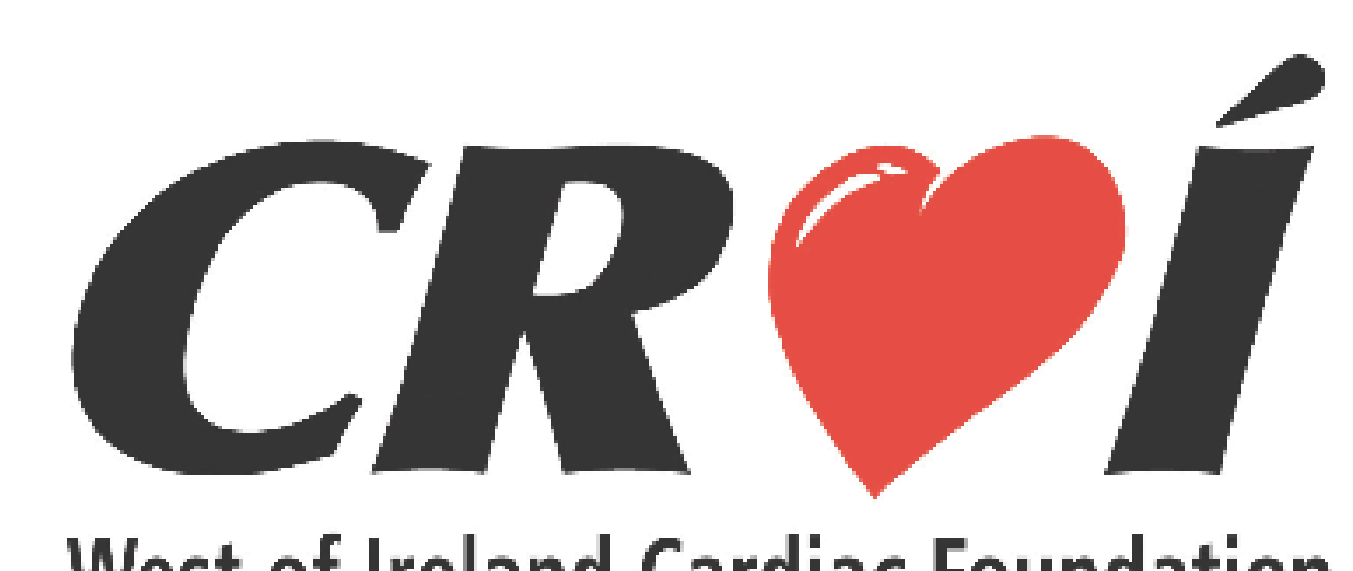

West of Ireland Cardiac Foundation 